\title{
Ganoderic acid B's influence towards the therapeutic window of trifluoperazine (TFP)
}

Jun Guo ${ }^{1, \#}$, Chenming $\mathrm{Ni}^{2, \#}$, Xiaoyang $\mathrm{Liu}^{3}$, Tao Liu ${ }^{4}$

1.Department of Neurology, Tangdu Hospital, the Fourth Military Medical University, Xi'an 710038, P.R. China 2. Laboratory of Pharmacology, Department of Pharmacy, Jinan Military General Hospital, Jinan 250031, P.R. China

3. Department of Hepatobiliary Surgery, Jinan Military General Hospital, Jinan 250031, P.R. China

4. Department of Dermatology, Tangdu Hospital, the Fourth Military Medical University, Xi'an 710038, P.R. China

\#These authors contributed equally to this work and should be considered as first authors

\section{Abstract}

Background: Ganoderic acid B is an important bioactive ingredient isolated from Ganoderma lucidum, and exhibits various pharmacological activities.

Aims: To investigate the influence of Ganoderic acid B towards the therapeutic window of trifluoperazine (TFP). Methods: In vitro human liver microsomes (HLMs) incubation system was used to determine the inhibition of Ganoderic acid B towards the glucuronidation of trifluoperazine (TFP).

Results: Ganoderic acid B exerted concentration-dependent inhibition towards the glucuronidation of TFP. Furthermore, Results: Ganoderic acid Bexe con (n) Dixo plot, indicating the competitive inhibition of Ganoderic acid B towards TFP glucuronidation. Through fitting the data using competitive nonlinear fitting equation, the inhibition kinetic parameter was calculated to be $56.7 \mathrm{uM}$.

Conclusion: All this data indicated the potential influence of Ganoderic acid B-containing herbs towards therapeutic window of TFP. Given that the glucuronidation reaction of TFP is the probe reaction of UGT1A4, the data obtained from the present study also indicated the potential influence of Ganoderic acid-containing herbs towards the therapeutic window of drugs mainly undergoing UGT1A4-mediated metabolism.

Keywords: Ganoderic acid B, trifluoperazine (TFP), UDP-glucuronosyltransferase (UGT) 1A4

DOI: http://dx.doi.org/10.4314/ahs.v15i1.20

\section{Introduction}

Ganoderma lucidum, a well-known traditional Chinese medicine, has been utilized for longevity and treatment of multiple diseases in Asia for many years ${ }^{1}$. Ganoderic acids, the oxygenated lanostane-type triterpenoids isolated from Ganoderma lucidum, have been reported to exhibit multiple biochemical and pharmacological activities, such as anti-tumor ${ }^{2,3}$, cardioprotective

\begin{tabular}{|l|}
\hline Corresponding author: \\
Tao Liu, \\
Department of Dermatology, \\
Tangdu hospital, the Fourth \\
Military Medical University, \\
No.569 Xinsi Road, Xi'an, 710038, \\
People's Republic of China \\
Email: ltfmmu@163.com \\
Tel: +86-29-84777729 \\
Fax: +86-29-84777729 \\
\hline
\end{tabular}

African Health Sciences Vol 15 Issue 1, March 2015 and liver protection activity ${ }^{4,5}$. The development of the compounds needs the adverse effects and in vivo elimination information, besides the pharmacologically therapeutic role.

Metabolic enzymes-catalyzed biotransformation process has been considered to be an important cause for the adverse effects of drugs. For example, cytochrome P450 (CYP) 2E1-mediated metabolic activation of acetaminophen has been regarded as the major reason for acetaminophen-induced acute liver failure ${ }^{6}$. Additionally, the inhibition of compounds towards the activity of drug-metabolizing enzymes (DMEs) is another reason for drugs-induced adverse effects. For example, the anti-tumor drug noscapine exhibits significant inhibitory effects towards CYP3A4 and CYP2C9-catalyzed warfarin metabolism, and then induces clinical noscapine-warfarin interaction ${ }^{7}$. For one of the most important phase II DMEs UDP- glucuronosyltransferases (UGTs), the activity inhibition will also induce the severe adverse effects. For example, HIV therapeutic drug indinavir inhibited UGT1A1-mediated bilirubin glucuronidation to induce the elevation of unconjugated bilirubin ${ }^{8}$.

Trifluoperazine (TFP) is a typical antipsychotic of the phenothiazine chemical class, and its major clinical application is to treat schizophrenia?. The adverse effects of TFP contain extrapyramidal reactions (e.g., Parkinson-like symptoms, dystonia, etc.), drowsiness, fatigue, muscular weakness, and hypotension.

According to the reports from Drugs.com website, a total of 1056 drugs can induce the drug-drug interaction (DDI) with TFP. TFP has relatively narrow therapeutic window, and major drug-metabolizing enzyme to contribute to the metabolism of TFP is UGT1A4. The present study aims to investigate the inhibition of Ganoderic Acid B towards the metabolism of TFP.

\section{Materials and Methods}

\section{Chemicals and Reagents}

Tris-HCl, 7-hydroxycoumarin, and uridine-5-diphosphoglucuronic acid (UDPGA) (trisodium salt) were obtained from Sigma-Aldrich (St. Louis, MO). Sigma-Aldrich also offers the trifluoperazine dihydrochloride for the research needs. Pooled human liver microsomes (HLMs) were prepared according to previous reports ${ }^{10,11}$.

Ganoderic Acid B's inhibition towards the glucuronidation of TFP

The formation of TFP glucuronide and the chromatography conditions were carried out as previously described $^{12}$. In brief, the incubation system $(200 \mathrm{uL})$ contains $50 \mathrm{mM}$ Tris- $\mathrm{HCl}(\mathrm{pH}=7.4), 25 \mathrm{ug} / \mathrm{mL}$ lamethicin (from Trichoderma viride), $0.1 \mathrm{mg} / \mathrm{ml}$ HLMs, $5 \mathrm{mM}$
$\mathrm{MgCl}$, $5 \mathrm{mM}$ UDPGA, and various concentrations TFP and Gaboderic Acid B. The incubation time was $20 \mathrm{~min}$, and the incubation temperature was $37^{\circ} \mathrm{C}$ The incubation conditions ensure the linear reaction of TFP glucuronidation. The reaction was terminated with the equal volume of methanol, and the aliquots $(10 \mathrm{uL})$ were used for analysis. The inhibition kinetic type was determined through Dixon plot (Reaction velocity versus the concentrations of Ganoderic acid B), and the inhibition kinetic parameters were calculated using the nonlinear regression equations as followed according to previous literatures13-15. $\mathrm{V}=(\operatorname{VmaxS}) /(\mathrm{Km}(1+\mathrm{I} /$ $\mathrm{Ki})+\mathrm{S})(1) \mathrm{V}=(\mathrm{VmaxS}) /(\mathrm{Km}+\mathrm{S})(1+\mathrm{I} / \mathrm{Ki})(2)$

The equations (1) and (2) were employed for competitive and noncompetitive fitting, respectively. The terms in the equations were defined as followed: $\mathrm{V}$ is the velocity, $\mathrm{S}$ is the concentration of substrate, $\mathrm{Km}$ is the metabolism kinetic parameter, I is the concentration of inhibitor, and $\mathrm{Ki}$ is the inhibition kinetic parameter.

Results

Multiple concentrations of Ganoderic acid B were used to screen the inhibition potential of Ganoderic acid B towards TFP glucuronidation, and the results showed that $0,20,40,60$ and $100 \mathrm{uM}$ of Ganoderic acid B inhibited the glucuronidation activity of TFP by $0,26.6 \%$, $44.1 \%, 52.9 \%$, and $66.9 \%$, respectively (Fig. 1).

Fig. 1 Concentration-dependent inhibition of Ganoderic Acid B towards the glucuronidation of trifluoperazine (TFP). The residul percentage of control activity was employed to represent the inhibition capability of Ganoderic acid B towards the glucuronidation activity of TFP. Two replicate experiments were carried out, and the values represent the mean value of duplicate experiment.
Figure 1

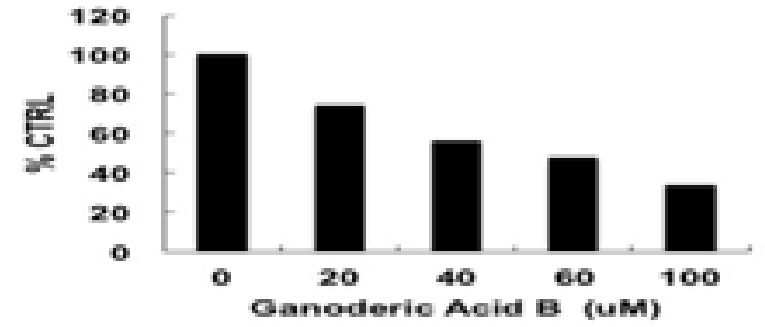


Furthermore, the Dixon plot using the 1 /reaction ve- fitting equation, the inhibition kinetic parameter (Ki) locity versus the concentrations of Ganoderic acid B was calculated to be $56.7 \mathrm{uM}$.

was drawn to determine the inhibition kinetic type, and

the intersection point was located into the second quad- Fig. 2 Determination of inhibition kinetic type using rant, indicating the competitive inhibition type (Fig. 2). the Dixon plot. The Dixon plot used the reaction veThrough fitting the data using competitive nonlinear locity versus the concentrations of Ganoderic acid B.

\section{Figure 2}

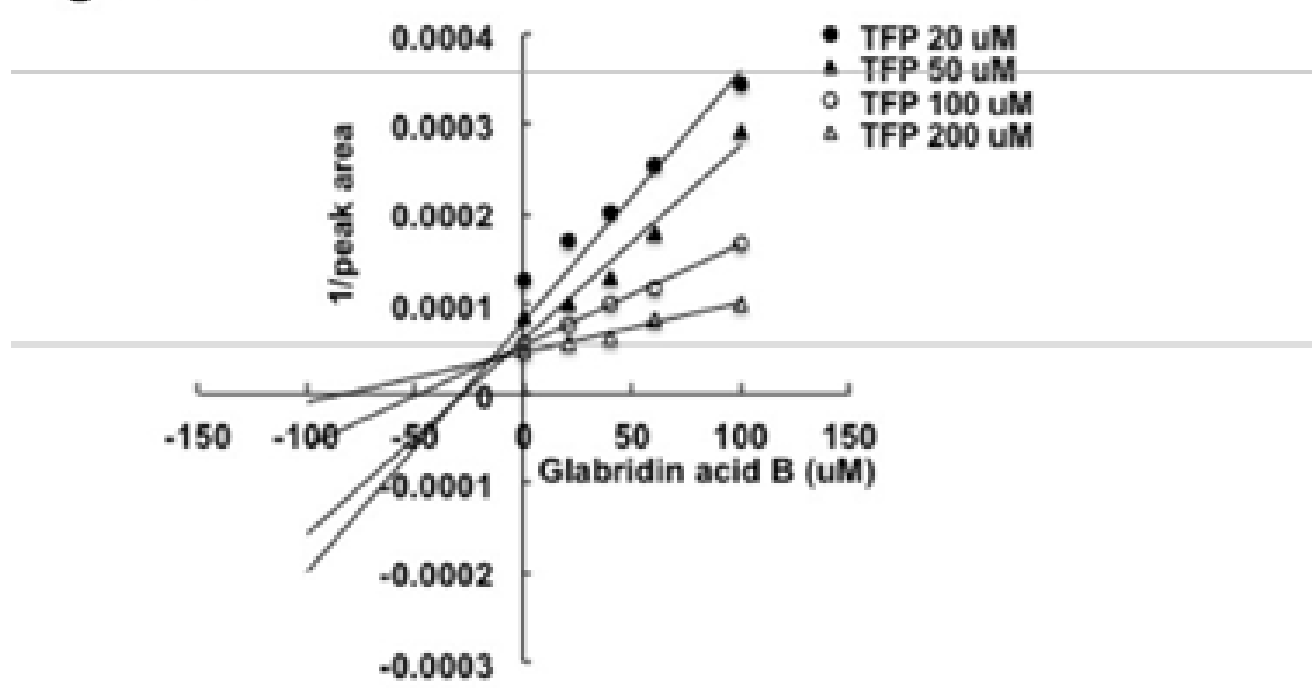

\section{Discussion}

In vitro incubation system is an useful tool to investigate the metabolic behavior of xenobiotics and metabolism-mediated adverse effects. Through adding different co-factors, the phase I and phase II metabolic pathways can be well separated, and the inhibition behavior towards the metabolism can be studied through co-incubation with another compound. The present study investigated the inhibition potential of Ganoderic acid B towards TFP glucuronidation, and the competitive inhibition of Ganoderic acid B was demonstrated, indicating the utilization risk when co-administration of Ganoderic acid B-containing herbs and TFP. Previous literatures have shown that other herbal components also exhibited inhibitory effects towards the glucuronidation of $\mathrm{TFP}^{16}$. Therefore, much attention should be paid to the clinical co-utilization of TFP and Ganoderic acid B-containing herbs.

It should be noted that the glucuronidation reaction of TFP has been widely employed as the probe reaction of UGT1A4. Many endogenous and xenobiotic Each data point represents the mean of the duplicate experiments.
$B$ are speculated to be the inhibitors of TFP glucuronidation, and will be investigated in the future.

\section{Acknowledgement}

This study was supported in part by National Natural Science Foundation of China (No. 30900772).

\section{References}

1. Guo X, Shen X, Long J, Han J, Che Q. Structural identification of the metabolites of ganoderic acid $\mathrm{B}$ from Ganoderma lucidum in rats based on liquid chromatography coupled with electrospray ionization hybrid ion trap and time-of-flight mass spectrometry. (2013) Biomed. Chromatogr. 2013; 27(9): 1177-1187. 2. Wu GS, Lu JJ, Guo JJ, Li YB, Tan W, Dang YY, Zhong ZF, Xu ZT, Chen XP, Wang YT. Ganoderic acid DM a natural triterpenoid, induces DNA damage, G1 cell cycle arrest and apoptosis in human breast cancer cells. Fitoterapia 2012; 83(2): 408-414

3. Li F, Wang Y, Wang X, Li J, Cui H, Niu M. Ganoderic acids suppress growth and angiogenesis by modulating the NF- $x \mathrm{~B}$ signaling pathway in breast cancer cells. Int J. Clin. Pharmacol. Ther. 2012; 50(10):712-721.

4. Kuok, Q.Y., C.Y. Yeh, B.C. Su, P.L. Hsu, H. Ni, M.Y Liu, et al. (2013) Mol. Nutr. Food Res. 57(10):1892-6. 5. Kuok QY1, Yeh CY, Su BC, Hsu PL, Ni H, Liu MY, Mo FE. The triterpenoids of Ganoderma tsugae prevent stress-induced myocardial injury in mice. Biotechnol. Lett. 2006; 28(11): 837-841

6. McGill MR, Jaeschke H. Metabolism and disposition of acetaminophen: recent advances in relation to hepatotoxicity and diagnosis. Pharm. Res. 2013; 30(9): 2174 2187.

7. Fang ZZ, Zhang YY, Ge GB, Huo H, Liang SC, Yang L. Time-dependent inhibition (TDI) of CYP3A4 and CYP2C9 by noscapine potentially explains clinical noscapine-warfarin interaction. Br. J. Clin. Pharmacol. 2010, 69(2): 193-199.

8. Zucker SD, Qin X, Rouster SD, Yu F, Green RM, Keshavan P, Feinberg J, Sherman KE. Mechanism of indinavir-induced hyperbilirubinemia. Proc. Natl. Acad. Sci. USA 2001; 99 (22):12671-12676.

9. Hempel C, Norenberg W, Sobottka H, Urban N, Nicke A, Fischer W, Schaefer M. The phenothiazine-class antipsychotic drugs prochlorperazine and trifluoperazine are potent allosteric modulators of the human P2X7 receptor. Neuropharmacology 2013; 75:365-379.

10. Song H, Tao Y, Zhang CZ, Zhou YD. Comparison of Cultured Hepatocytes Results with Human Liver
Microsomes (HLMs) for the Glucuronidation Elimination of Zidovudine (AZT). Lat. Am. J. Pharm. 2013; 32 (4): 606-609.

11. Fang ZZ, Krausz KW, Nagaoka K, Tanaka N, Gowda K, Amin SG, Perdew GH, Gonzalez FJ. In vivo effects of the pure aryl hydrocarbon receptor antagonist GNF-351 after oral administration are limited to the gastrointestinal tract. Br. J. Pharmacol. 2014; 171(7):17351746.

12. Uchaipichat V, Mackenzie PI, Elliot DJ, Miners JO, Selectivity of substrate (trifluoperazine) and inhibitor (amitriptyline, androsterone, canrenoic acid, hecogenin, phenylbutazone, quinidine, quinine, and sulfinpyrazone) "probes" for human udp- glucuronosyltransferases. Drug Metab. Dispos. 2006; 34: 449-456.

13. Zhao HD, Bao GQ, He XL, Wu T, Wang CG, Wang SZ, et al. Strong Inhibition of UDP-Glucuronosyltransferase (UGT) 1A1 by Levothyroxine Indicates the Potential UGT-Inhibition Based Adverse Effect of Levothyroxine. Lat. Am. J. Pharm. 2012; 31(5): 761-763. 14. Shan LN, Shi XB. Strong Inhibition Capability of Wogonin towards UDP-Glucuronosyltransferase (UGT) 1A1 and 1A3. Lat. Am. J. Pharm. 2013; 32(5): 741-744.

5. Yang S, Li M, Ji CB, Liang YC, Qin LC, Du YF. Prediction of Potential Herb-Drug Interaction Based on the Inhibition of Specific Intestinal Drug-Metabolizing Enzyme (DME) by Wogonin. Lat. Am. J. Pharm. 2013; 32(4): 613-615.

16. Ma HY, Sun DX, Cao YF, Ai CZ, Qu YQ, Hu CM, et al., Herb-drug interaction prediction based on the high specific inhibition of andrographolide derivatives towards UDP-glucuronosyltransferase (UGT) 2B7. Toxicol. Appl. Pharmacol. 2014; 277(1): 86-94.

17. Wang Z, Wong T, Hashizume T, Dickmann LZ, Scian M, Koszewski NZ, et al. Human UGT1A4 and UGT1A3 conjugate 25-hydroxyvitamin D3: metabolite structure, kinetics, inducibility, and interindividual variability. Endocrinology 2014; 155(6):2052-2063

8. Laverdiere I, Caron P, Harvey M, Levesque E, Guillemette C. In vitro investigation of human UDP-glucuronosyltransferase isoforms responsible for tacrolimus glucuronidation: predominant contribution of UGT1 A4. Drug Metab. Dispos. 2011; 39(7):1127-1130.

19. Green MD, Bishop WP, Tephly TR. Expressed human UGT1.4 protein catalyzes the formation of quaternary ammonium-linked glucuronides. Drug Metab. Dispos. 1995; 23: 299-302. erazine (TFP) was reported in the present study, as indicated by the in vitro inhibition of Ganoderic acid B towards the glucuronidation of TFP. Additionally, many compounds with similar structures with Ganoderic acid 2000; 143-161.

African Health Sciences Vol 15 Issue 1, March 2015 